\title{
PSYCHOPHYSIOLOGICAL MEASURES OF DRIVER DISTRACTION AND WORKLOAD WHILE INTOXICATED
}

\author{
Michael E. Rakauskas, Nicholas J. Ward \\ HumanFIRST Program \\ ITS Institute, University of Minnesota \\ Minneapolis, Minnesota, USA \\ E-mail: mickr@me.umn.edu, nicw@me.umn.edu
}

\author{
Edward Bernat, Meredith Cadwallader, Christopher Patrick \\ Department of Psychology \\ University of Minnesota \\ Minneapolis, Minnesota, USA \\ Dick de Waard \\ University of Groningen \\ The Netherlands
}

\begin{abstract}
Summary: The crash risk associated with cell phone use while driving is a contentious issue. Many states are introducing Advanced Traveler Information Systems (ATIS) that may be accessed with cell phones while driving (e.g., 511 Traveler Information Services). In these contexts, there is a need for relevant research to determine the risk of cell phone use. This study compared driver performance while conversing on a hands-free cell phone to conditions of operating common in-vehicle controls (e.g., radio, fan, air conditioning) and alcohol intoxication (BAC 0.08). In addition, the study examined the combined effects of being distracted and being intoxicated given that there may be a higher risk of a crash if the driver engages in a combination of risk factors. During simulated traffic scenarios, resource allocation was assessed through an eventrelated potential (ERP) novelty oddball paradigm. Intoxicated drivers were less attentive to all stimuli and drivers engaged in secondary tasks had weaker responses to unexpected novel sounds in brain regions associated with evaluative processing. Drivers conversing on the cell phone and in-vehicle tasks while sober had lower accuracy during the target tone task than intoxicated drivers not completing any secondary task.
\end{abstract}

\section{INTRODUCTION}

There is considerable debate regarding cell phones as a risk factor in traffic crashes. Specifically, there is insufficient knowledge about the relative risk of cell phone use while driving compared to other existing secondary tasks drivers may perform in the vehicle. Now that there are many states intending to introduce traveler information systems (e.g., 511 Traveler Information Services) that may be accessed with cell phones while driving, there is an even greater need for relevant research to determine the risk of secondary task distractions including cell phone use while driving. 
This study had two primary purposes. First, the study used measures of psychophysiology indicative of driver distraction and workload to quantify the impairment of hands-free cell phone conversations relative to the benchmarks of (1) common in-vehicle tasks (adjust radio or climate controls) and (2) legal alcohol intoxication. Second, the study used these same measures to quantify the interaction effect of combining alcohol with distraction tasks (cell phone conversation, in-vehicle tasks). In this manner, psychophysiological measures were used to quantify the (relative) impairment effects associated with cell phone use.

\section{METHODS}

Forty-eight male participants were examined in total, twenty-four of whom participated in the ERP methodology. Participants had to be at least 21 years old $(M=22.3$ years $)$, live within a distance to the university (or on a bus route) that permitted them to walk to and from the experiment location on campus, and showed no evidence of alcoholism as assessed by the CAGE alcoholism screening instrument (Mayfield, McLeod, \& Hall, 1974).

\section{Driving Simulator}

The study used the Virtual Environment for Simulation in Transportation Research (VESTR). This immersive, motion-base driving simulator is linked to a full-sized Saturn vehicle with realistic operational controls and instrumentation. The visual scene is projected with a highresolution (2.5 arc-minutes per pixel) five-channel 210-degree forward field of view with 50degree rear projected field of view and side mirrors comprised of color LCD panels. Auditory feedback and haptic feedback were provided by a 3D surround audio system, subwoofer, car body vibration, force feedback steering, and a three-axis electric motion system (roll, pitch, zaxis). The vehicle was equipped with a Compaq iPAQ 3635 Pocket PC mounted on the dashboard near the driver to display the in-vehicle task instructions. Cell phone conversations were communicated through a simulated after-market hands-free speaker system. The simulated driving environment was comprised of a realistic rural highway with one 2-way-STOP intersection based on roads in northern Minnesota.

\section{Secondary Task Conditions}

In addition to baseline driving without any distraction, two types of secondary task were developed to represent different combinations of resource allocation to information input, processing, and output response in relation to primary driving task resources. The cell phone task was an audio input and verbal output task, while the in-vehicle task was a visual input and manual output task. Thus the two tasks required different modes of attention and response. However, each task appeared to be equally demanding as evident by a similar number of observed task attempts and reported subjective mental effort $(\mathrm{p}>.05)$.

Cell Phone (Hands-Free) Condition. The conversation tasks were based on questions from the Rosenbaum Verbal Cognitive Test Battery (Waugh, Glumm, Kilduff, Tauson, Smyth, C., and Pillalamarri, 2000) intended to represent different levels of mental demand by measuring judgment and flexible thinking in a limited response time. Questions required the driver to repeat a sentence, solve a puzzle (both requiring just a single response), or talk on a stated topic (requiring twenty or more seconds of response and assisted by the experimenter). 
In-Vehicle Condition. A Compaq iPAQ mounted on the dashboard was used to display images of the radio and HVAC system (a visual display was used because audio instruction would represent the same resource demands of listening to a cell phone conversation). The screen showed an image of a system setting to signify that the participant was to copy the setting depicted on the actual vehicle controls. The two types of task were selecting a button (HVAC or $\mathrm{CD}$ track) or changing a knob position (HVAC only). Each picture shown was preceded by one second of flashing to alert the driver to a new task instruction.

\section{Psychophysiological Measures}

We used an event-related potential (ERP) novelty oddball auditory paradigm. Participants listened to a series of standard and target tones ( $80 \%$ and $10 \%$, respectively) and were to respond with a left foot pedal when they heard the target tone of a higher pitch. They would also infrequently hear task-irrelevant (novel) sounds (10\% of trials). Physiological measurements to the oddball task employed electroencephalogram (EEG) recordings using a 40-channel Neuroscan NuAmp Quick Cap. Surface electrodes placed directly on the scalp directly gauge the brain's activity during a task. Within the overall recording, time-locked cortical ERPs to the novel and target sound stimuli were extracted for analysis. ERP responses to stimuli of the same type (targets and novels) are averaged together and contain a series of negative and positive signal elements generally referred to as components (see Figure 2). Particularly relevant is the amplitude of a positive signal, peaking around $250-500 \mathrm{~ms}$ after the onset of the stimulus of interest, known as the P300. Studies have shown P300 amplitude to be inversely related to stimulus probability (Squires, Wickens, Squires, and Donchin, 1976), and therefore, target tones and novel sounds maintain the largest P300 amplitudes in the oddball task.

P300 amplitude has proven an effective measure of workload when employed as a secondary task. As an increasing amount of processing resources are dedicated to the primary task, the ability to formulate a pattern of expectancy to the target and novel sounds is proportionately interrupted, and consequently, the P300 amplitude to these sounds is proportionately attenuated (see Wickens, Isreal, and Donchin, 1977). Janssen \& Gaillard (1984) were able to discriminate task load within three distinct road environments by measuring P300 amplitude during an oddball task within a car-driving scenario.

ERP data was epoched from 500ms before stimulus delivery to 1000 ms post-stimulus. P300 measures were taken as the peak amplitude between 250 and $500 \mathrm{~ms}$, relative to an equivalent $150 \mathrm{~ms}$ of baseline activity. A correction procedure was applied to adjust for vertical and horizontal ocular artifacts (Semlitsch, Anderer, Schuster, and Presslich, 1986). Trials on which amplitude in the $1000 \mathrm{~ms}$ post-stimulus window exceeded $+/-100 \mu \mathrm{V}$ were excluded from analysis. Analyzed data was taken from all electrodes, with emphasis on the frontal (Fz), central $(\mathrm{Cz})$ and parietal sites $(\mathrm{Pz})$ that are distributed along the anterior to posterior midline of the brain. Past research has localized the novel P300 (often referred to as P3a) as a maximally frontocentral (Fz-Cz) effect, and the target P300 (often referred to as P3b) as a maximally parietal (Pz) effect (Comerchero \& Polich, 1999).

An additional between-subjects factor was physiological response in the form of inter-beat interval (IBI). Electrocardiogram (ECG) readings were recorded from the left and right forearms just below the elbows with two $1 \mathrm{~cm}$ Med Associates $\mathrm{Ag}-\mathrm{AgCl}$ electrodes filled with electrolyte 
paste to record heart rate IBI. From the ECG, R-peaks were detected with 1 millisecond accuracy and these were time-stamped written to file. After artifact correction power spectral analyses were computed with aid of the CARSPAN (CARdiovascular SPectral ANalysis) programme. To reduce inter-individual differences a natural logarithmic $(\mathrm{Ln})$ transformation was performed on the power spectra data (Van Roon, 1998). A lower IBI (an increase in heart rate) is indicative for increased controlled information processing and increased mental effort.

\section{Dependent Measures}

Psychophysiological measures of mental processes were quantified through performance measures and metrics of attention resource allocation.

- The performance measures consisted of:

- Reaction Time (RT) to the target tones [milliseconds]

- Target tone accuracy [percentage of correct responses]

- The attention resource allocation measures consisted of:

- Novel sounds P300

- Heart rate inter beat-intervals (IBI) [milliseconds]

There were no significant effects for P300 response to the target tones at the $\mathrm{Fz}$ (frontal), $\mathrm{Cz}$ (central), and $\mathrm{Pz}$ (parietal, rear) electrodes; thus, they are not explored here.

\section{Design and Analysis}

Participants drank either a placebo containing no alcohol (Control group) or an alcoholic beverage to produce a BAC of 0.08 (Alcohol group; BAC $\mathrm{M}=.073$ ). Thus, BAC Group was a between-subjects factor. The overall experimental design was a 3 (Task Condition: Baseline, Cell Phone, In-Vehicle) x 2 (BAC Group: Alcohol, Control) mixed-factor design.

The performance data were analyzed with a mixed-factor design ANOVA (with Huynh-Feldt correction for potential sphericity). The task by group simple effects were examined with a oneway Friedman's $\left(\mathrm{X}_{\mathrm{F}}^{2}\right)$ non-parametric test across the task levels separately for each BAC group (sober and drunk groups). All follow-up tests were completed using the Wilcoxon $\left(\mathrm{Z}_{\mathrm{W}}\right)$ nonparametric signed-ranks test. "Benchmark tests" to compare drunk, non-distracted drivers with sober, distracted drivers used Mann-Whitney $\left(Z_{U}\right)$ non-parametric paired tests. All data was screened for outliers in this design and a significance level of $p<.05$ was used for all statistics. The ERP resource allocation data used comparison of composite P300 response waves from all electrodes (for an example and full explanation, see Figure 2 in the Results section).

\section{RESULTS}

\section{Performance Measures}

Reaction Time. There was a significant main effect of Task Condition for response time to target tones $[\mathrm{F}(2,36)=19.00, \mathrm{p}<.001]$. As shown in Figure 1a, response time was significantly slower 
during the in-vehicle task $\left[\mathrm{W}_{\mathrm{z}}=3.70, \mathrm{p}<.001\right]$ and cell phone $\left[\mathrm{W}_{\mathrm{z}}=3.74, \mathrm{p}<.001\right]$ conditions compared to the baseline driving. Reaction time during both secondary task conditions was similar. However, benchmark testing indicated that the reaction time while sober and engaged in cell phone conversations $(\mathrm{M}=825 \mathrm{~ms})$ was marginally slower $\left[\mathrm{U}_{\mathrm{z}}=1.85, \mathrm{p}<.10\right]$ than while drunk and driving with no other secondary tasks $(\mathrm{M}=683 \mathrm{~ms})$.

Target Tone Accuracy. There was a significant main effect of Task Condition for response accuracy to detect target tones $[\mathrm{F}(2,36)=9.67, \mathrm{p}<.001]$. As shown in Figure $1 \mathrm{~b}$, response accuracy was significantly lower during the in-vehicle task $\left[\mathrm{W}_{\mathrm{z}}=2.13, \mathrm{p}<.05\right]$ and cell phone $\left[\mathrm{W}_{\mathrm{z}}=3.38, \mathrm{p}<.001\right]$ conditions compared to the baseline driving. Response accuracy during the cell phone condition was also lower than for the in-vehicle task condition $\left[\mathrm{W}_{\mathrm{z}}=2.49, \mathrm{p}<.01\right]$. Indeed, benchmark testing indicated that the response accuracy while sober and engaged in cell phone conversations $(\mathrm{M}=81 \%)$ was marginally lower $\left[\mathrm{U}_{\mathrm{z}}=3.15, \mathrm{p}<.01\right]$ than drunk and driving with no other secondary tasks $(\mathrm{M}=96 \%)$.

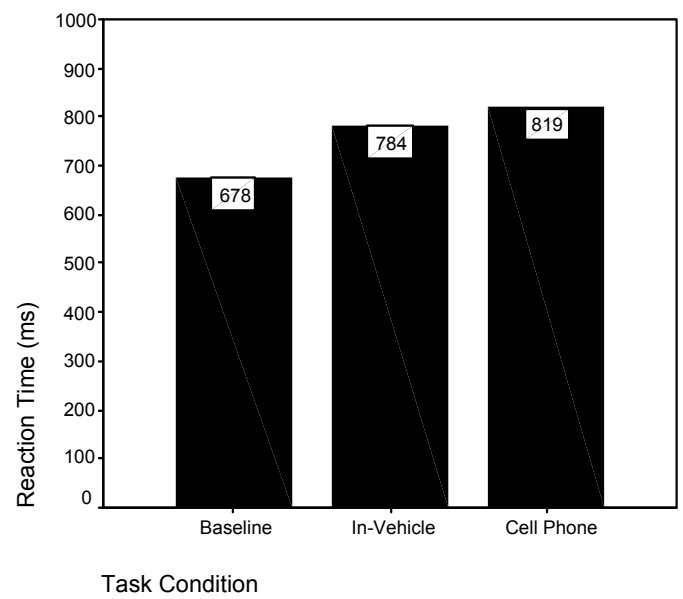

a.

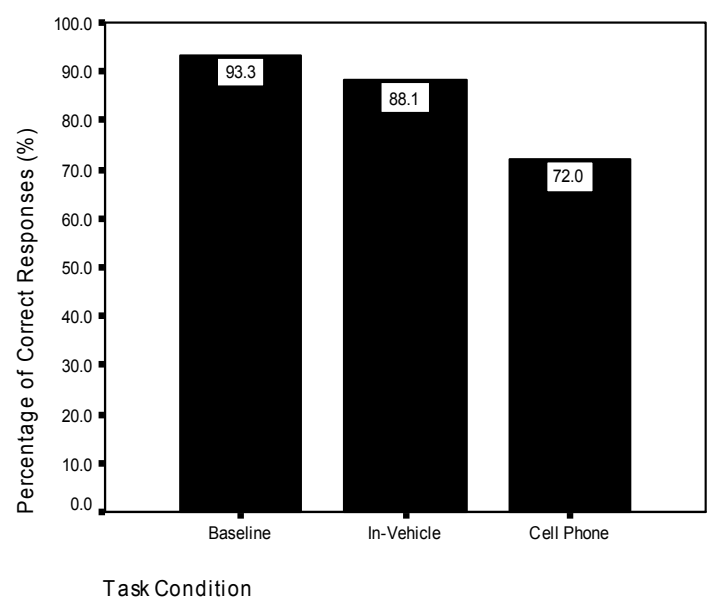

b.

Figure 1. Target tone (a) Reaction time as a function of task condition, and (b) Detection accuracy

\section{Attention Resource Allocation}

Novel Sounds P300. Intoxication was found to cause a significant reduction in amplification of the novelty-P300 signal in intoxicated drivers, as compared to sober drivers. On the left of Figure 2, composite P300 waves for sober and intoxicated drivers are shown in comparison. To the right, the left-most head diagram shows the differences between particular processing areas in the brain, where darker colors indicate larger differences between the Sober and Intoxicated P300 composite waves. The right-most head model shows which differences are statistically significant $(\mathrm{p}<.05)$ in white.

The head models show that intoxicated drivers had a reduced response to novel sounds as evident by the significant difference in the central and parietal (rear) functioning of the brain. This suggests that intoxicated drivers spent less of their mental capacity on an evaluative 
response to novel (unexpected) sounds. Thus, they can be said to be cognitively impaired by alcohol at a physiological level.

?ำ
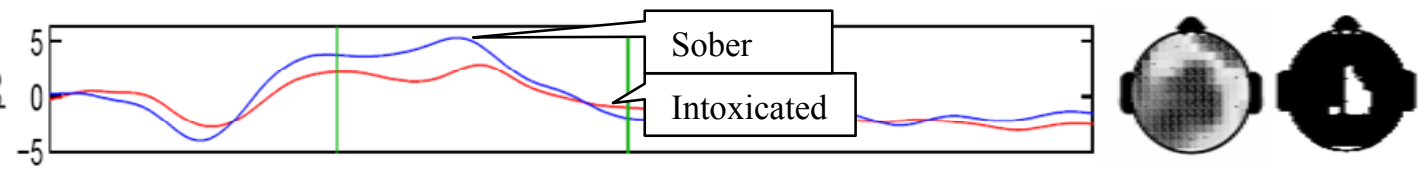

Figure 2. ERP of P300 response to novel sounds between sober and intoxicated drivers along with head models showing location and significance of wave differences

Analysis of the P300 response to the novel sounds at all locations showed that the cell phone condition showed the largest impairment to processing in the parietal region (Figure 3) as compared to the baseline condition. This suggests that drivers completing the cell phone condition were more cognitively impaired, especially in terms of evaluating sudden and unexpected stimuli.
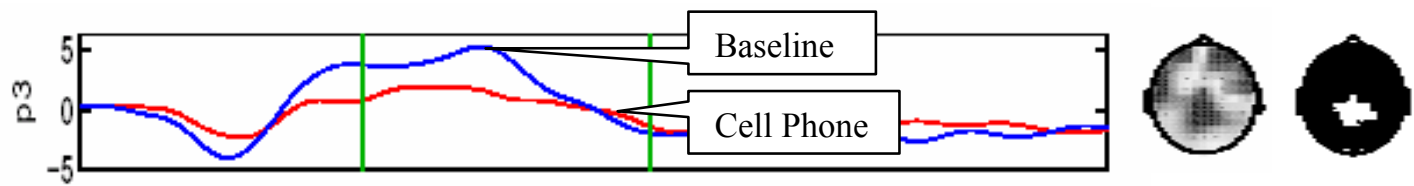

Figure 3. ERP of novelty-P300 response for drivers' responses during cell phone and baseline conditions

A less significant impairment effect was seen when comparing the in-vehicle task alone to baseline driving (Figure 4). This may suggest that drivers were more cognitively impaired at a physiological level during the cell phone condition than while completing in-vehicle tasks as compared to baseline driving.

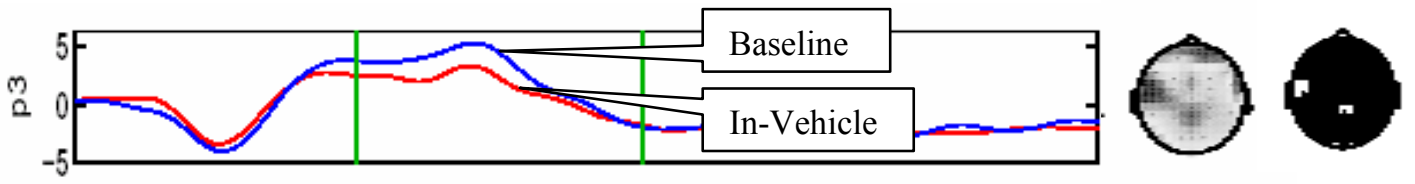

Figure 4. ERP of novelty-P300 response for drivers' responses during baseline and the in-vehicle conditions

Heart Rate IBI. A resting heart rate was used as a significant covariate $[\mathrm{F}(1,18)=86.6, \mathrm{p}<.001]$. There was a significant main effect for Task Condition $[\mathrm{F}(2,36)=4.93, \mathrm{p}<.01]$. As shown in Figure 5, physiological indications of workload were significantly higher during the hands-free cell phone condition than during baseline driving without any secondary task $\left[\mathrm{W}_{\mathrm{z}}=2.48\right.$, $\mathrm{p}<.01]$. Moreover, the measured workload in the cell phone condition was also marginally higher than during the in-vehicle task condition $\left[\mathrm{W}_{\mathrm{z}}=1.76, \mathrm{p}<.08\right]$. There was no significant difference in workload between the baseline and in-vehicle task conditions. 


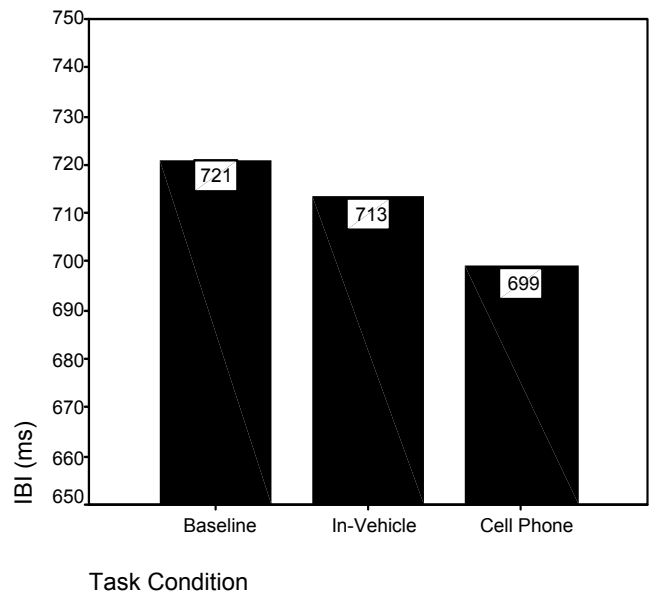

Figure 5. Heart rate IBI (physiological workload response; covariate adjusted) during car following scenario as a function of task condition

\section{CONCLUSIONS}

Heart rate results indicated higher levels of inferred workload during both secondary tasks, with the highest workload observed while driving and engaged in cell phone conversations. The ERP oddball paradigm was validated by confirming previous research that alcohol reduces attention (Burns et al., 2002; Strayer et al., 2003), while also showing little evidence that the paradigm itself impaired performance. Both response performance and topographic mapping of brain activity in relation to $\mathrm{P} 300$ indicated reduced processing of external stimuli, especially information that was not expected. Indeed, there was some compelling evidence that distracted drivers may be more impaired on some of these measures of attention capacity than drunk drivers. These results may imply that drivers distracted by secondary tasks such as cell phones may incur a high workload to manage these dual tasks such that they have fewer resources available to correctly evaluate unexpected events. Secondary tasks may significantly impact safety to the extent that drivers in these conditions are unable to accurately evaluate the hazard imposed by objects with which they may unexpectedly interact.

\section{REFRENCES}

Burns, P., Parkes, A., Burton, S., Smith, R., Burch, D. (2002). How dangerous is driving with a mobile phone? Benchmarking the impairment to alcohol. Draft Report. Transport Research Laboratory (TRL), Crowthorne, Berkshire, RG45, 6AU, UK, http://www.trl.co.uk.

Janssen, W.H. \& Gaillard, A.W.K. (1984). Task load and stress on the road: Preliminaries to a model of route choice. Report Institute for Perception TNO, IZF 1984, C-10.

Mayfield, D., McLeod, G., Hall, P. (1974). The CAGE questionnaire: validation of a new alcoholism screening instrument. American Journal of Psychiatry, 131(10), 1121-1123.

Semlitsch, H. V., Anderer, P., Schuster, P., and Presslich, O. (1986). A solution for reliable and valid reduction of ocular artifacts applied to the P300 ERP. Psychophysiology, 1986, 23, 695703). 
Strayer, D. L., Drews, F. A., Crouch, D. J. (2003). Fatal Distraction? A comparison of the cellphone driver and the drunk driver. Proceedings of the Second International Driving Symposium on Human Factors in Driver Assessment, Training, and Vehicle Design. Park City, UT, USA. July 21-24, 2003, pp. 25-30.

Squires, K.C., Wickens, C., Squires, N.K. \& Donchin, E. (1976). The effect of stimulus sequence on the waveform of the cortical event-related potential. Science, 193, 1142-1146.

Van Roon, A.M. (1998). Short-term cardiovascular effects of mental tasks. Physiology, experiments and computer simulation. $\mathrm{PhD}$ thesis, University of Groningen. Groningen, The Netherlands.

Waugh, J., Glumm, M., Kilduff, P., Tauson, R., Smyth, C., Pillalamarri, R. (2000). Cognitive workload while driving and talking on a cellular phone or to a passenger. International Ergonomics Association Conference, San Diego, USA. [As cited in Burns et al., 2002.].

Wickens, C.D., Isreal, J. \& Donchin, E. (1977). The event-related cortical potential as an index of task workload. Proceedings of the Twenty-First Annual Meeting of the Human Factors Society, San Francisco. 